\title{
Oridonin Sensitizes Hepatocellular Carcinoma to the Anticancer Effect of Sorafenib by Targeting the Akt Pathway
}

This article was published in the following Dove Press journal: Cancer Management and Research

\author{
Xuguang $\mathrm{Li}^{1,2}$ \\ Weirun Chen ${ }^{2}$ \\ Kaihang $\mathrm{Liu}^{3}$ \\ Sheng Zhang ${ }^{2}$ \\ Ru Yang ${ }^{4}$ \\ Kairui $\mathrm{Liu}^{2}$ \\ Dateng $\mathrm{Li}^{5}$ \\ Youxing Huang $\mathbb{D}^{2}$
}

'Department of General Surgery, The First Affiliated Hospital of Jinan University, Guangzhou, Guangdong Province, People's Republic of China; ${ }^{2}$ Department of Abdominal Surgery, The Second Affiliated Hospital of Guangzhou University of Chinese Medicine,

Guangzhou, Guangdong Province, People's Republic of China; ${ }^{3}$ Department of General Practice, The People's Hospital of Longhua, Shenzhen, Guangdong Province, People's Republic of China; ${ }^{4}$ Department of General Practice, Women \& Children Health Institute Futian Shenzhen, Shenzhen, Guangdong Province, People's Republic of China; ${ }^{5}$ Department of Statistical Science, Southern Methodist University, Dallas, TX 75275, USA
Correspondence: Youxing Huang Department of Abdominal Surgery, The Second Affiliated Hospital of Guangzhou University of Chinese Medicine, No. II I Dade Road, Guangzhou, Guangdong Province, People's Republic of China

Tel +86-|363225544|

$\mathrm{Fax}+86-20-39318790$

Email waiqike7@।63.com
Background: Oridonin is the core bioactive component of Rabdosia rubescens, a traditional Chinese herbal medicine used in the treatment of hepatoma. Sorafenib, a targeted therapeutic agent for advanced hepatocellular carcinoma (HCC), has recently been shown to exert limited clinical effects. However, few studies have focused on the synergistic effect of these two drugs on hepatocellular carcinoma.

Methods: We treated different HCC cell lines with different concentrations of oridonin and sorafenib and assessed the viability by using MTT assays and examined proliferation, migration, invasion and apoptosis after cotreatment of HepG2 cells with $20 \mu \mathrm{M}$ oridonin and $5 \mu \mathrm{M}$ sorafenib via colony formation assays, Transwell assays and flow cytometry. Regulatory effects were measured by Western blotting. The in vivo synergistic effect was confirmed through xenograft tumor models, and tumor tissues were analyzed by immunohistochemistry.

Results: The inhibitory effects of oridonin and sorafenib cotreatment on HCC cells were stronger than those of either drug alone. In addition, combined treatment with the two drugs synergistically inhibited epithelial-mesenchymal transition and the Akt pathway but not NF$\kappa \mathrm{B}$ or MAPK signaling. Akt phosphorylation by SC79 reversed the inhibitory effects of the combined treatment. Synergistic inhibition was equally observed in vivo.

Conclusion: Oridonin combined with sorafenib synergistically inhibited proliferation, migration, invasion, and epithelial-mesenchymal transition and induced apoptosis by targeting the Akt pathway but not NF-kB or MAPK signaling.

Keywords: oridonin, sorafenib, HCC, Akt pathway

\section{Introduction}

Hepatocellular carcinoma (HCC) is the third leading cause of cancer-associated mortality worldwide. ${ }^{1}$ Researchers have made tremendous efforts to understand the biological mechanisms that restrain HCC development. Multiple treatment strategies, including surgical resection, ablation, TACE, targeted therapeutic drugs and liver transplantation, have been applied to different stages of HCC to improve prognosis. However, the overall 5-year survival rate remains unsatisfactory. ${ }^{2}$ Major challenges in treating HCC are the limitations of surgical management for patients with advanced $\mathrm{HCC}$, high recurrence after surgery and the lack of efficient drugs with mild side effects. Sorafenib is the first targeted therapeutic agent available for advanced HCC and exerts antitumor effects by inhibiting tumor cell proliferation, preventing angiogenesis and inducing cell apoptosis. ${ }^{3}$ However, emerging evidence has revealed the limited 
clinical effects of sorafenib because of the development of resistance. For example, Andrea Casadei-Gardini and his colleagues validated a simple scoring system to individuate predictive factors of sorafenib response and found that chronic treatment with metformin could results in resistance to sorafenib. ${ }^{4-6}$ Although great efforts have been done to improve the clinical efficacy, the median survival increased by sorafenib was still no more than 3 months for patients with advanced $\mathrm{HCC}^{3-7}$

Traditional Chinese herbal medicine has played a vital role in fighting varieties of cancer types for millennia. Rabdosia rubescens (Hemsl.), a medicinal herb, has long been used in China as an anti-inflammatory and antitumor agent. $^{8}$ Oridonin, the major bioactive component of Rabdosia rubescens, shows favorable therapeutic effects against many cancer types, such as breast cancer, ovarian cancer, colon cancer, osteosarcoma, gastric cancer, lung cancer, esophageal squamous cell carcinoma and hepatocellular carcinoma. ${ }^{9}$ In addition, oridonin shows synergistic anticancer effects when combined with other treatments. For example, cotreatment with oridonin and cisplatin inhibited the transcriptional activity of $\mathrm{NF}-\kappa \mathrm{B}$ and induced apoptosis in $\mathrm{HCC},{ }^{10}$ oridonin enhanced radiation-induced cell death in lung cancer, ${ }^{11}$ and oridonin intensified the toxicity of $5-\mathrm{FU}$ in renal carcinoma. ${ }^{12}$ However, the synergistic effect of oridonin and sorafenib remains unclear, although both drugs are effective in treating HCC.

In this study, we found that the combination of oridonin and sorafenib attenuated the viability of HCC cells in a dose-dependent manner. In addition, cotreatment with the two drugs inhibited proliferation, migration, and invasion and induced apoptosis in vitro and mitigated tumor growth in vivo. Moreover, combined treatment inhibited epithelial-mesenchymal transition (EMT) and alleviated Akt phosphorylation but not NF- $\mathrm{B}$ or MAPK signaling. Furthermore, we demonstrated that the synergistic antitumor effect of oridonin and sorafenib was modulated by the Akt pathway.

\section{Materials and Methods Cell Culture and Treatment}

Human hepatocellular carcinoma cell lines, including SMMC-7721, HepG2, Bel-7402, and Huh7 cells, were obtained from the Type Culture Collection of the Chinese Academy of Sciences (Shanghai, China). The cells were cultured in Dulbecco's modified Eagle's medium (DMEM) (GibcoBRL Life Technologies, Grand Island) with 10\% fetal bovine serum (FBS) (Biological Industries, Israel) and $1 \%$ penicillin-streptomycinneomycin (GibcoBRL Life Technologies, Grand Island) at the permissive temperature $\left(37^{\circ} \mathrm{C}\right)$. Oridonin (Selleck, USA), sorafenib (Bayer, Germany) and SC79 (Selleck, USA) were dissolved in dimethyl sulfoxide (DMSO) (MPBIO; USA), stored at $-20^{\circ} \mathrm{C}$ and diluted to the target concentrations with complete medium before use.

\section{MTT Assay}

A 3-(4,5-dimethylthiazol-2-yl)-2,5-diphenyltetrazolium bromide (MTT) kit (Beyotime, China) was used to analyze cell viability. The four HCC cell lines were inoculated in 96-well plates (1500 cells per well) and treated with orido$\operatorname{nin}(0,10,20$ and $30 \mu \mathrm{M})$, sorafenib $(0,2.5,5$ and $10 \mu \mathrm{M})$ or sorafenib combined with oridonin at the indicated concentrations for $48 \mathrm{~h}$. Thereafter, $20 \mu \mathrm{L}$ of MTT solution was added to each well and incubated at $37^{\circ} \mathrm{C}$ for $4 \mathrm{~h}$. Then, the supernatants were removed, and $150 \mu \mathrm{L}$ of DMSO was added to each well. Cell viability was detected by measuring the absorbance at $570 \mathrm{~nm}$ with an automated microplate reader (Thermo Fisher Scientific, USA).

\section{Colony Formation Assay}

HepG2 cells were plated in six-well plates at a density of $5 \times 10^{2}$ cells/well and then stimulated with $20 \mu \mathrm{M}$ oridonin, 5 $\mu \mathrm{M}$ sorafenib, $20 \mu \mathrm{M}$ oridonin combined with $5 \mu \mathrm{M}$ sorafenib and $0.2 \%$ DMSO as a negative control. The cells were observed daily under an optical microscope (Olympus, Japan). Ten days later, cell culture was terminated, and 4\% paraformaldehyde was added to each well for $30 \mathrm{~min}$ to fix the cells. Then, the fixed cells were stained with $1 \%$ crystal violet solution (Solarbio, Beijing, China). Colonies of more than 50 adherent cells were counted.

\section{Apoptosis Assay}

We performed a flow cytometric assay to evaluate cell apoptosis. After the administration of $20 \mu \mathrm{M}$ oridonin, 5 $\mu \mathrm{M}$ sorafenib, $20 \mu \mathrm{M}$ oridonin combined with $5 \mu \mathrm{M}$ sorafenib and $0.2 \%$ DMSO as a negative control for $48 \mathrm{~h}$, the cells were fixed in $80 \%$ ethanol after collection and stored at $4{ }^{\circ} \mathrm{C}$ overnight. After the cells were washed with PBS, RNase A and propidium iodide were added. Then, the cells were incubated for $20 \mathrm{~min}$ at $4^{\circ} \mathrm{C}$ in the dark. Cells were suspended at a density of $1 \times 10^{6}$ cells per milliliter in buffer containing FITC-conjugated Annexin V/PI (Keygen, Nanjing, China) before analysis via flow 
cytometry. The proportion of apoptotic cells to total cells was calculated.

\section{Transwell Migration and Invasion Assay} HepG2 cells were suspended in serum-free DMEM at a density of $1 \times 10^{5} / \mathrm{mL}$. The Transwell inserts were precoated with fibronectin for migration assays and $\mathrm{BD}^{\mathrm{TM}}$ Matrigel (BD, USA) for invasion assays. The abovementioned cell suspension $(100 \mu \mathrm{L})$ was added to the upper chamber of the Transwell inserts. Next, $600 \mu \mathrm{L}$ of DMEM with $10 \%$ FBS was added to the lower chamber to act as a chemoattractant. Oridonin $(20 \mu \mathrm{M})$, sorafenib $(5 \mu \mathrm{M})$, oridonin $(20 \mu \mathrm{M})$ combined with sorafenib $(5 \mu \mathrm{M})$ and DMSO (0.2\% DMSO) as a negative control were added to both the upper and lower chambers. After incubation for $24 \mathrm{~h}$ for the migration assay and $48 \mathrm{~h}$ for the invasion assay, the cells on the lower insert surface were fixed with $4 \%$ paraformaldehyde and stained with $1 \%$ crystal violet (Beyotime, China). The number of cells was counted in three different microscopic fields of three independent inserts.

\section{Western Blotting}

HepG2 cells were treated with $20 \mu \mathrm{M}$ oridonin, $5 \mu \mathrm{M}$ sorafenib, $20 \mu \mathrm{M}$ oridonin combined with $5 \mu \mathrm{M}$ sorafenib and $0.2 \%$ DMSO for $48 \mathrm{~h}$, lysed in RIPA buffer (Beyotime, China) and centrifuged. A BCA protein assay kit (Thermo, USA) was used to measure the protein concentration. Samples were diluted in 5× SDS loading buffer and separated by $10 \%$ or $12 \%$ SDS-PAGE before being transferred to polyvinylidene fluoride (PVDF) membranes (Millipore). The PVDF membranes were blocked in 5\% nonfat dry milk at room temperature, incubated with appropriate primary antibodies (1:1000) (Cell Signaling Technology, USA), including GAPDH, PCNA, Bcl-2, Bax, E-cadherin, Vimentin, Snail, cleaved PARP, cleaved Caspase-3, p-Akt, Akt, p-p65, p65, p-JNK, JNK, p-p38, p38, p-ERK, ERK, and thereafter incubated with appropriate HRP-conjugated secondary antibodies (1:3000, Beyotime, China). The immunoreactive bands were visualized using an ECL Western blot kit (CW0049C, CWBIO). The protein expression levels were standardized to GAPDH and were assessed by densitometry using ImageJ (National Institutes of Health, USA).

\section{Animals and Treatments}

In vivo experiments were performed in the Animal Laboratory Center of Jinan University and all animals were used according to institutional guidelines (Institute of laboratory animal science, Jinan University). Ethics and legal approval (No. SYXK2012-0117) was obtained from the Committee for Experimental Animal Studies of Jinan University prior to the commencement of the study. Fourto six-week-old male nude (BALB/c nu/nu) mice were subcutaneously injected with $100 \mu \mathrm{L}$ of serum-free medium containing HepG2 cells $\left(1 \times 10^{7}\right.$ cells $)$ in the left abdomen. Seven days later, the mice were randomly divided into four groups and were fed water, oridonin (30 mg/kg), sorafenib $(20 \mathrm{mg} / \mathrm{kg})$ or oridonin $(30 \mathrm{mg} / \mathrm{kg})$ combined with sorafenib $(20 \mathrm{mg} / \mathrm{kg})$ daily. We measured and recorded the tumor volumes with a digital caliper each week, and four weeks after injection, the mice were anesthetized with 3\% isoflurane (Sigma, USA) and sacrificed by cervical dislocation. The tumors were excised, photographed and weighed.

\section{Immunohistochemistry}

Fresh tumor tissues from xenograft animals were fixed in formalin for $48 \mathrm{~h}$ before being prepared into paraformaldehyde-fixed paraffin-embedded $4 \mu \mathrm{m}$-thick sections. After deparaffinization in xylene, rehydration in ethanol and rinsing in PBS, the sections were treated with TE (10 mM Tris/1 mM EDTA, [pH 9.0]) at subboiling temperatures and subsequently incubated with $3 \%$ hydrogen peroxide after being cooled to room temperature. The sections were blocked with $200 \mu \mathrm{L}$ of normal goat serum (ZSGB-BIO, China) for $1 \mathrm{~h}$ at $37^{\circ}$ $\mathrm{C}$ and then incubated with $200 \mu \mathrm{L}$ of primary antibodies, including p-Akt (Abcam, USA), Ki67 (Abcam, USA), Vimentin (Abcam, USA), and Bcl-2 (Abcam, USA), at a dilution of 1:100 overnight at $4^{\circ} \mathrm{C}$. After being washed with PBS, the tissue sections were incubated with diluted streptavidin-peroxidase HRP conjugates and then stained with hematoxylin for $3 \mathrm{~min}$ before analysis under a microscope. The staining results were blindly assessed by two researchers according to the previously described scoring criteria. ${ }^{13}$

\section{Statistical Analysis}

All results were obtained from three independent experiments and were repeated three times in each individual experiment under the same conditions. All data are expressed as the mean \pm standard deviation (SD). Differences among groups were compared using GraphPad Prism (GraphPad Software, USA) by Student's t-tests and 
one-way ANOVA. Differences were considered statistically significant when $\mathrm{p}$ values were less than $0.05(\mathrm{p}<0.05)$.

\section{Results}

\section{Synergistic Inhibition of Hepatocellular Carcinoma Cell Lines by Oridonin and}

\section{Sorafenib}

We first investigated the synergistic effects of oridonin and sorafenib on HCC cell lines. HepG2, Bel-7402, SMMC7721, and Huh7 cells were treated with increasing concentrations of oridonin and sorafenib for $48 \mathrm{~h}$, and analyzed by MTT assays. As shown in Figure 1, oridonin alone significantly inhibited the viability of the different HCC cell lines in a concentration-dependent manner. When oridonin and sorafenib were used in combination, the inhibitory effect on HCC cells was stronger than that of either drug alone, and the inhibitory effect was significantly enhanced with increasing concentrations of each drug. These results indicated that oridonin and sorafenib synergistically inhibited the viability of HCC cells. We selected HepG2 cells and used oridonin at a dose of $20 \mu \mathrm{M}$ and sorafenib at a dose of $5 \mu \mathrm{M}$ for further investigation.

\section{Cotreatment with Oridonin and}

Sorafenib Synergistically Inhibited

\section{Proliferation and Induced Apoptosis}

We further explored the effects of oridonin and sorafenib on the proliferation and apoptosis of HCC cells. As observed under an optical microscope, at $48 \mathrm{~h}$ after drug treatment, the cell morphology of the combined treatment group changed conspicuously, and the number of detached apoptotic cells significantly increased (Figure 2C). We examined the effects of different treatments on cell proliferation by colony formation assays. As shown in Figure 2A, oridonin or sorafenib alone could reduce the colony counts of HepG2 cells. Oridonin alone inhibited the proliferation of HepG2 cells to $80.92 \% \pm 2.55 \%$ compared with that of the negative control, while sorafenib alone suppressed cell proliferation to $66.30 \%$ $\pm 3.38 \%$. When oridonin and sorafenib were used in combination, the colony counts were only $29.28 \% \pm 4.46 \%$ compared with those of the control group, which were significantly less than those of single drug treatments. Similar results were also obtained when we examined apoptosis by flow cytometry (Figure 2B). The apoptosis ratios of the control, oridonin, sorafenib and cotreatment groups were
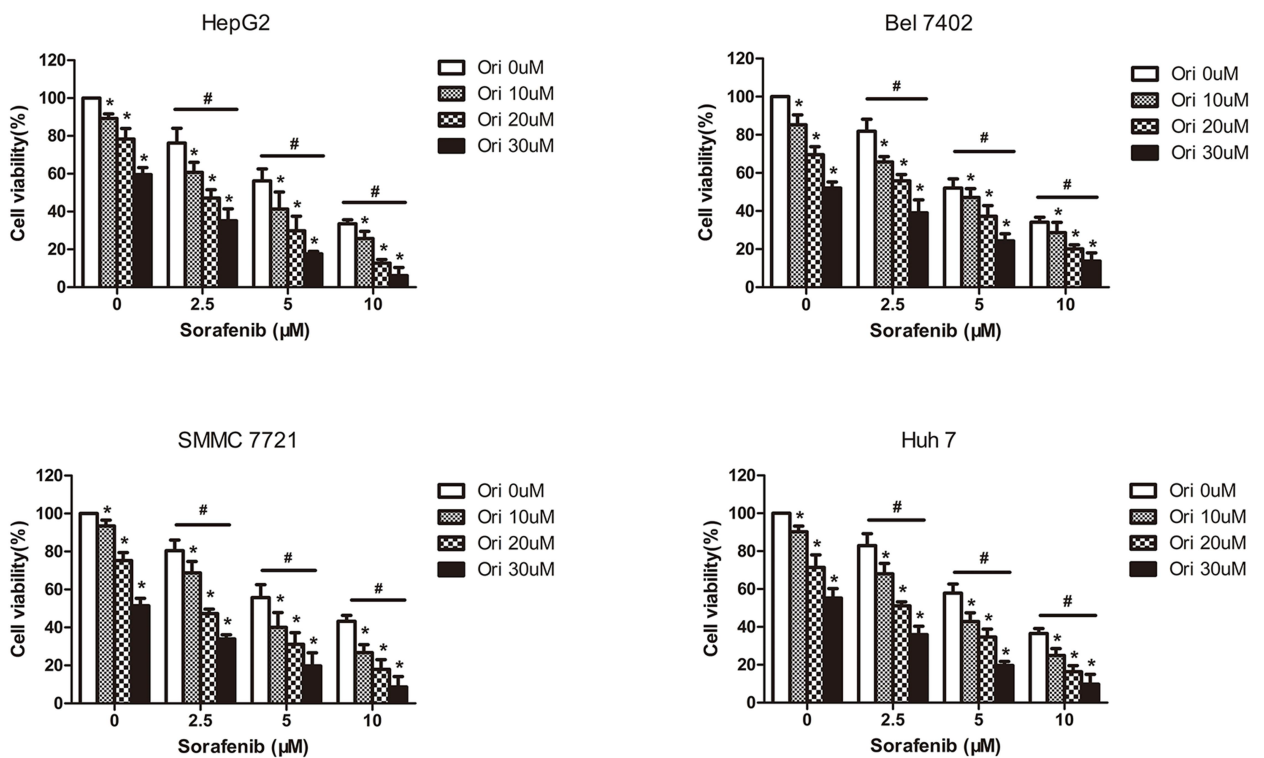

Figure I Cytotoxicity of the combination of different doses of oridonin and sorafenib on HCC cell lines. The viability of HepG2, Bel-7402, SMMC-772I and Huh7 cells was analyzed by MTT assays after treatment with different concentrations of sorafenib and oridonin for $48 \mathrm{~h}$. (The data represent the mean \pm SD of three independent experiments. *Indicates $p<0.05$ compared with $0 \mu \mathrm{M}$ oridonin; ${ }^{*}$ Indicates $\mathrm{p}<0.05$ compared with $0 \mu \mathrm{M}$ sorafenib; Ori indicates oridonin; Sor indicates sorafenib; Ori + Sor indicates combined oridonin and sorafenib treatment). 
A
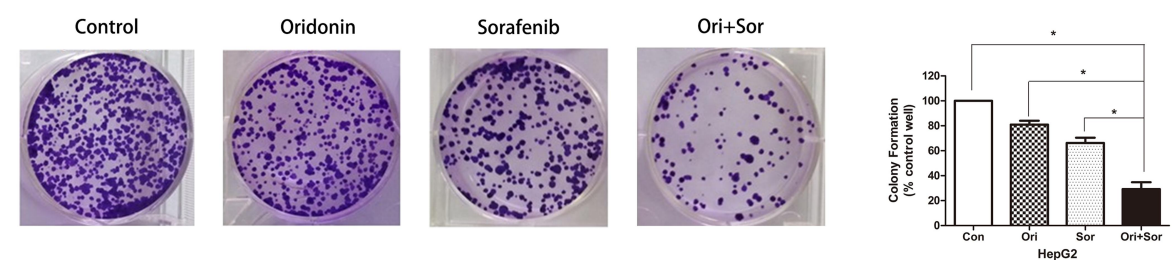

B
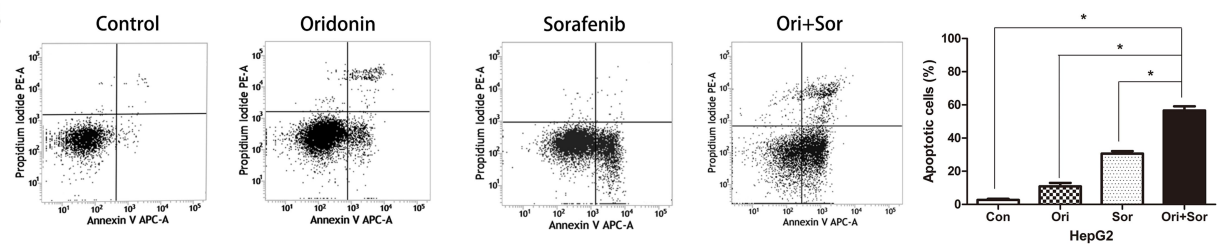

C
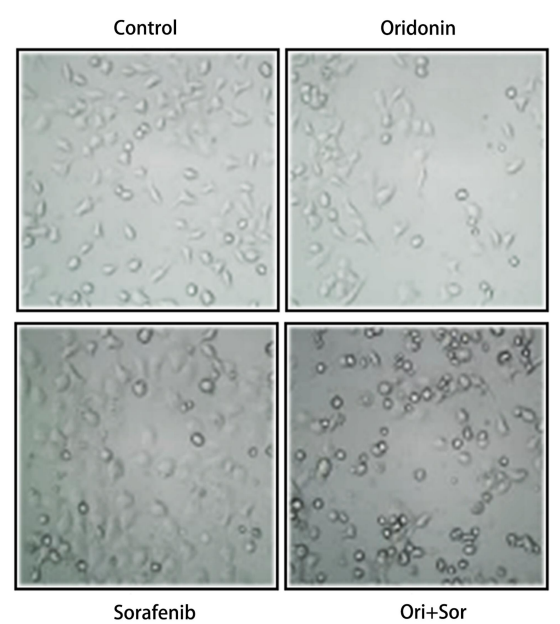

D

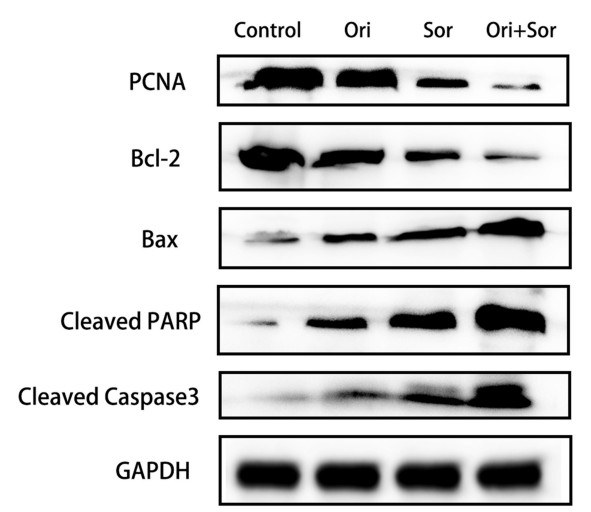

Figure 2 Cotreatment with oridonin and sorafenib synergistically inhibited proliferation and induced apoptosis in HepG2 cells. (A) The proliferation of HepG2 cells was measured by colony formation assays after treatment with oridonin, sorafenib and oridonin combined with sorafenib. (B) Flow cytometry showed enhanced apoptosis induction in HepG2 cells after combined treatment with oridonin and sorafenib. (C) Representative micrographs of the cellular morphological signatures after the treatments. (D) The expression of PCNA, Bcl-2, Bax, cleaved PARP and cleaved caspase 3 was analyzed by Western blotting. (The data represent the mean \pm SD of three independent experiments. *Indicates $p<0.05$ compared with the combination group; Ori indicates oridonin; Sor indicates sorafenib; Ori + Sor indicates combined oridonin and sorafenib treatment).

$2.73 \% \pm 0.55 \%, 10.90 \% \pm 1.66 \%, 30.60 \% \pm 1.27 \%$ and $56.65 \%$ $\pm 1.99 \%$, respectively, and the differences were statistically significant. In addition, we measured the expression of PCNA, Bax, Bcl-2, cleaved PARP and cleaved Caspase-3 by Western blotting (Figure 2D). The expression of PCNA and Bcl-2 was notably decreased, while the expression of Bax, cleaved PARP and cleaved caspase-3 (apoptosis-related proteins) was markedly increased. These results indicated that oridonin or sorafenib treatment alone inhibited HepG2 cell proliferation and induced apoptosis, while cotreatment was superior to the effect of either drug alone.

\section{Combined Treatment with Oridonin and Sorafenib Further Suppressed Metastasis and Epithelial-Mesenchymal Transition}

To assess the synergistic effect of oridonin and sorafenib on metastasis, we performed Transwell assays. As shown in
Figure 3A, the number of migrating cells after cotreatment with oridonin and sorafenib was significantly less than that after treatment with a single drug. The number of migrating cells was $272 \pm 8.99$ for the control group, $214 \pm 11.28$ for the oridonin group, $139 \pm 9.06$ for the sorafenib group and 62 \pm 13.97 for the cotreatment group. Similar results were observed in the cell invasion assay (Figure 3B), in which $238 \pm 13.92$ were observed for the control group, $171 \pm 10.22$ were observed for the oridonin group, $103 \pm 8.29$ were observed for the sorafenib group and $32 \pm 6.22$ were observed for the cotreatment group, and the differences were statistically significant. Because epithelial-mesenchymal transition plays a pivotal role in cancer cell migration and invasion, we investigated whether combined treatment with oridonin and sorafenib regulated epithelial-mesenchymal transition in liver cancer cells by measuring the expression profile of EMT markers (Figure 3C). Combined treatment significantly 

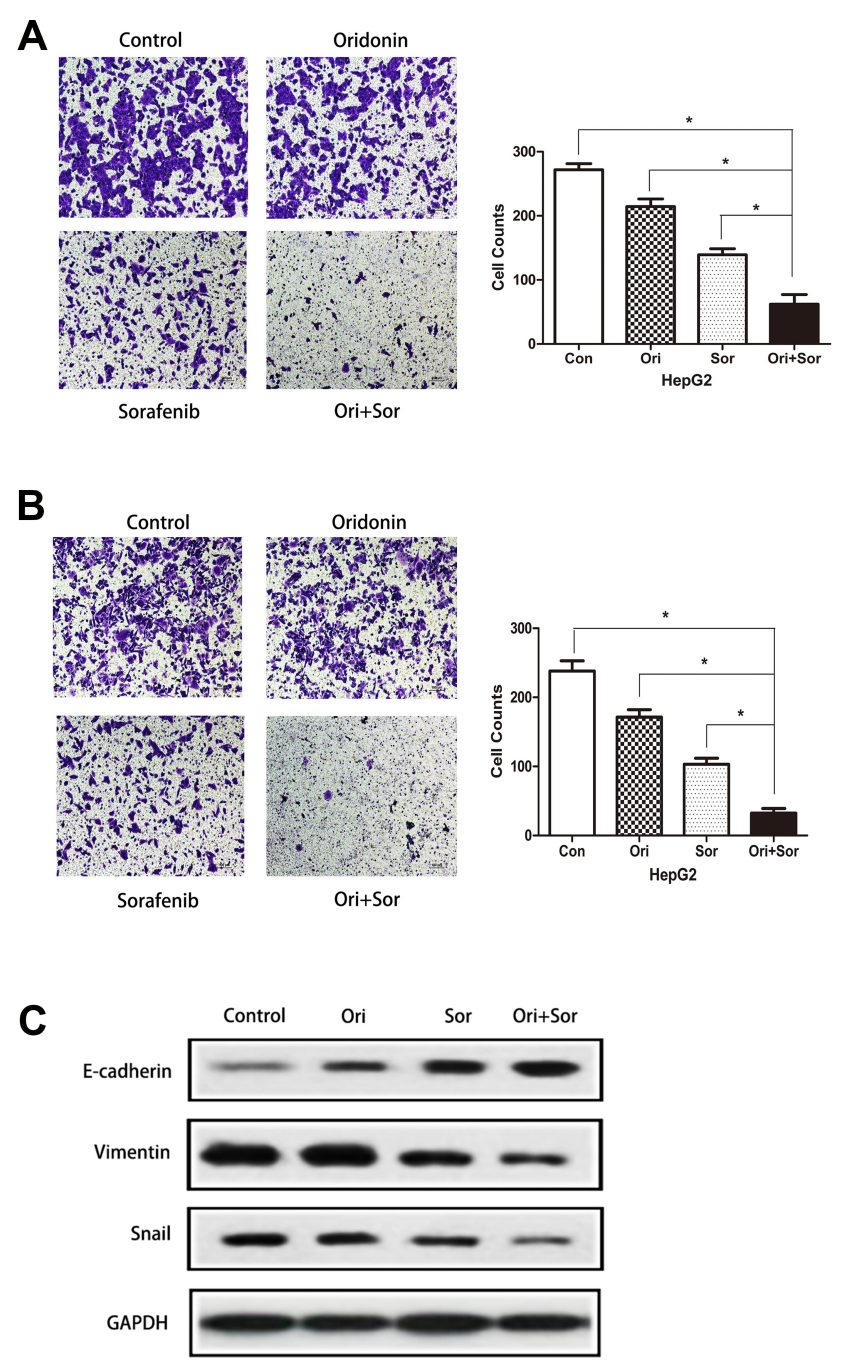

Figure 3 Combined treatment with oridonin and sorafenib suppressed metastasis and regulated EMT in HepG2 cells. (A) Transwell assays showed that oridonin combined with sorafenib inhibited the migration of HepG2 cells. (B) Representative images show that oridonin and sorafenib synergistically suppressed the invasion of HepG2 cells. (C) The levels of E-cadherin, vimentin and Snail were measured by Western blotting. (The data represent the mean \pm SD of three independent experiments. *Indicates $p<0.05$ compared with the combination group; Ori indicates oridonin; Sor indicates sorafenib; Ori + Sor indicates combined oridonin and sorafenib treatment).

enhanced the expression level of the epithelial protein E-cadherin and the transcription factor Snail and reduced the mesenchymal protein Vimentin compared with those of single drug treatments. These results suggested that cotreatment with oridonin and sorafenib suppressed HCC cell metastasis and epithelial-mesenchymal transition.

\section{Oridonin and Sorafenib Synergistically Mitigated Activation of the Akt Pathway but Not the MAPK or NF- $\kappa B$ Pathway}

A variety of signaling pathways, such as the Akt, NF-kB, JNK, p38, and Erk signaling pathways, are associated with the progression of hepatocellular carcinoma, and some of these pathways are targets of sorafenib. Therefore, we explored the regulation of their phosphorylation after treatment with oridonin, sorafenib and oridonin combined with sorafenib (Figure 4). The results suggested that after the combined treatment with oridonin and sorafenib, Akt phosphorylation was decreased significantly compared with that of single drug treatments. However, the activation of the NF- $\mathrm{kB}$, JNK, p38 and Erk signaling pathways changed little after cotreatment compared with that after treatment with sorafenib alone. These results indicate that the Akt pathway participates in the inhibitory effects caused by cotreatment with oridonin and sorafenib.

\section{The Akt Pathway Played a Pivotal Role in the Anticancer Effect of Combined Oridonin and Sorafenib Treatment}

To further explore the association between the Akt pathway and the inhibitory effects of cotreatment, we treated cells with SC79 to activate the Akt pathway after combined treatment with the two drugs. We first measured the expression levels of proteins associated with proliferation, apoptosis and EMT. As shown in Figure 5A, SC79 treatment restored Akt phosphorylation, which could reverse the expression of proliferation proteins, antiapoptotic proteins, apoptotic proteins and EMT markers. Cotreatment with oridonin and sorafenib downregulated PCNA, Bcl-2, Vimentin, and Snail and upregulated Bax, cleaved PARP, cleaved caspase-3, and E-cadherin, which were restored by Akt reactivation. Similar results were observed when we performed colony formation assays, flow cytometry and Transwell assays (Figure 5B-D). Cells cotreated with the two drugs under Akt activation conditions showed stronger proliferative, antiapoptotic, migratory and invasive abilities than those of cells without Akt activation. These results suggested that the synergistic inhibitory effect of combined oridonin and sorafenib treatment was modulated by the Akt pathway in HCC.

\section{Oridonin and Sorafenib Synergistically Exerted a Potent Anticancer Effect Against $\mathrm{HCC}$ in vivo}

To further confirm the synergistic inhibitory effect of oridonin and sorafenib in vivo, xenograft tumor models were prepared with HepG2 cells. Tumor growth in the combined treatment group was significantly mitigated compared with that of the other groups. Moreover, the tumor weight in the oridonin group was $85.9 \%$ of that in 


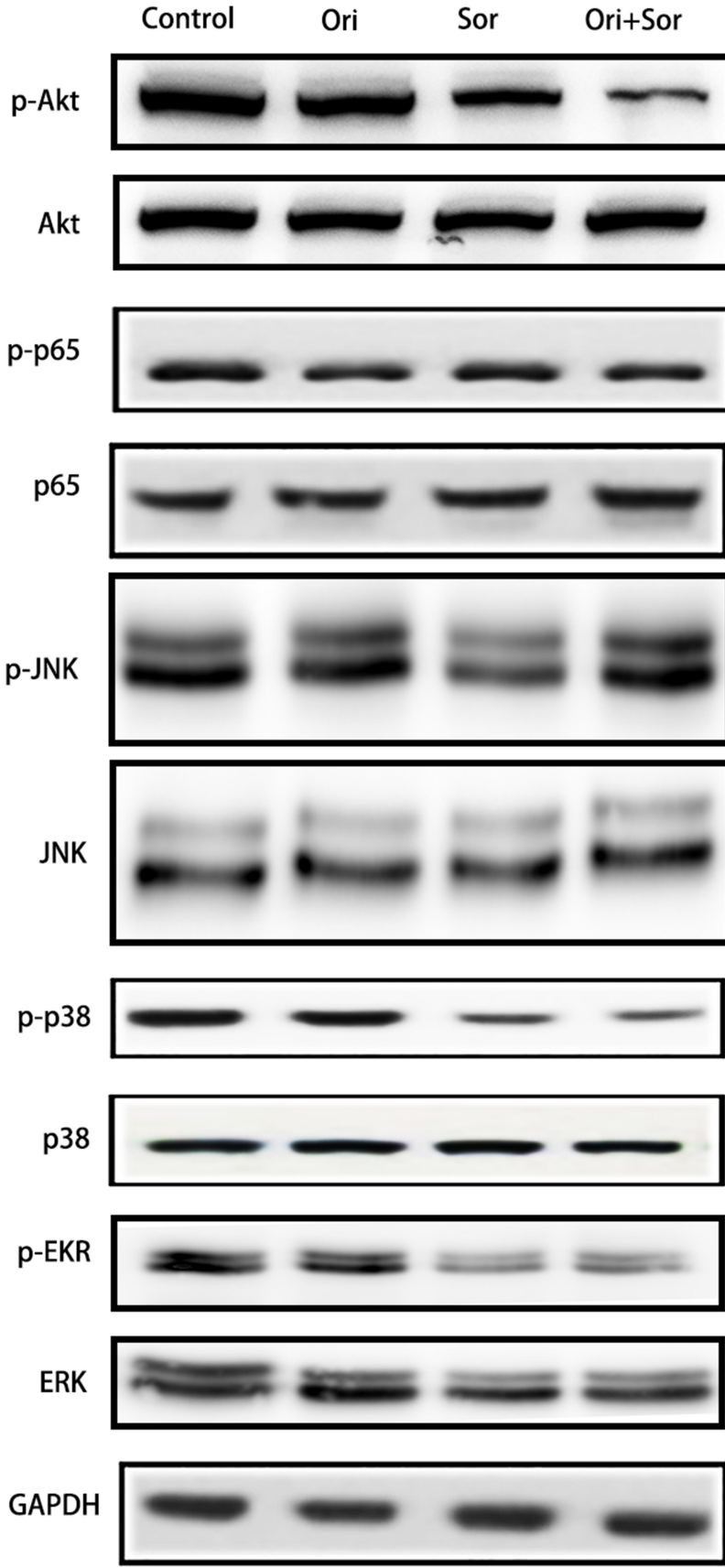

Figure 4 Cotreatment with oridonin and sorafenib mitigated activation of the Akt pathway but not the MAPK or NF-kB pathway. Activation levels of Akt, p65, JNK, $\mathrm{p} 38$, and Erk signaling pathway proteins in each group were examined by Western blotting. (Ori indicates oridonin; Sor indicates sorafenib; Ori + Sor indicates combined oridonin and sorafenib treatment).

the control group, and in the sorafenib group, it was $54.9 \%$, while it was $24.3 \%$ in the combined treatment group (Figure 6A). Subsequently, we measured the expression of proteins related to the Akt pathway, proliferation, apoptosis and EMT to confirm the in vitro results. Tumor sections from the xenograft tumor models were stained for p-Akt, Ki67, Vimentin and Bcl-2. The results were similar to the in vitro results. The combination of oridonin and sorafenib significantly decreased the expression of p-Akt, Ki67, Vimentin and Bcl-2 (Figure 6B). Taken together, these results suggest that oridonin combined with sorafenib synergistically inhibits HCC progression via the Akt pathway.

\section{Discussion}

Rabdosia rubescens is an effective traditional Chinese medicine for the treatment of hepatoma. ${ }^{9}$ As its main component, oridonin has been proven to inhibit the progression of HCC. The potential mechanism may be associated with the regulation of mitochondrial ROS and ES stress to induce cell apoptosis. For example, it has been reported that oridonin activates oxidative stress pathways leading to HepG2 cell death, Hsp70-1 is a key protective protein in oridonin-mediated oxidative stress, ${ }^{14}$ oridonin modulates ER stress and $\alpha-\mathrm{CP} 1$, leading to proliferation inhibition and apoptosis induction in HCC cells, ${ }^{9}$ and oridonin-induced HCC cell apoptosis is mediated by mitochondrial ROS through PARP activation, p53, and the MAPK pathway. ${ }^{15,16}$ In addition, considering that oridonin could effectively inhibit $\mathrm{HCC}$, many researchers have extracted oridonin derivatives, which have also been proven to have significant inhibitory effects on the progression of HCC. ${ }^{17,18}$ Therefore, oridonin might be a therapeutic candidate for the treatment of HCC. In this study, similar results were demonstrated by measuring the viability of HepG2, Bel-7402, Huh7 and SMMC-7721 cells after treatment with different concentrations of oridonin. We found that oridonin could inhibit the proliferation, migration, and invasion of $\mathrm{HCC}$ cells and induce apoptosis. Oridonin alone induces mesenchymalepithelial transformation in HCC cells and inhibits the

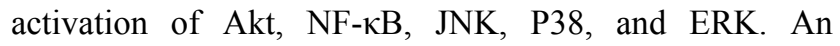
in vivo study also confirmed that oral intake of oridonin alone could inhibit tumor growth in mice and downregulate proliferation, metastasis, and antiapoptotic proteins.

Sorafenib is the first targeted drug to effectively increase the survival of patients with advanced $\mathrm{HCC}$, but the improvement in survival time is not satisfactory due to drug resistance. ${ }^{3,7}$ In addition, due to various adverse reactions, such as hand, foot and skin reactions, diarrhea, hypertension, fatigue, and hair loss, drug discontinuance is sometimes inevitable, and some of the patients cannot benefit from sorafenib treatment. ${ }^{19}$ Combined treatment with antitumor drugs contributes to increasing the curative effect and reducing the side effects through the synergistic 


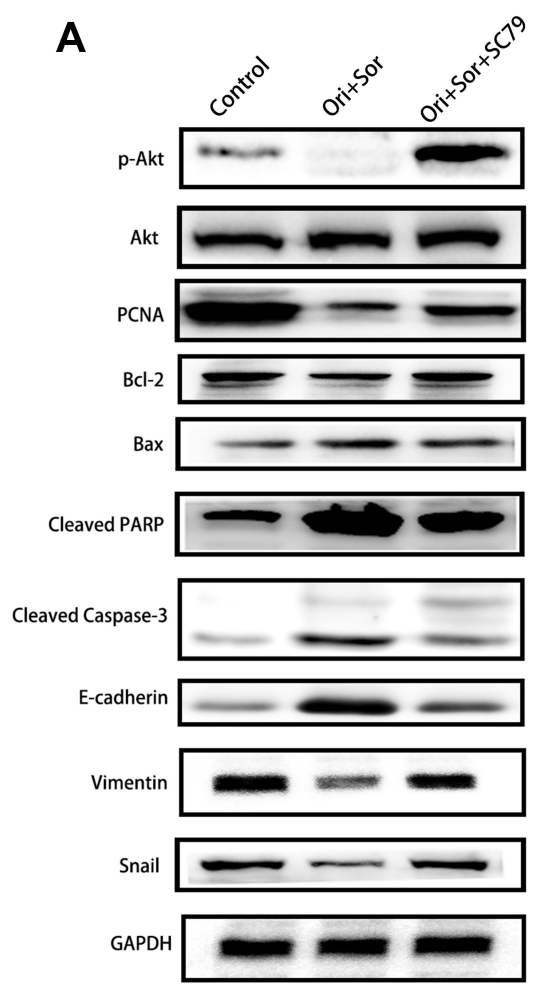

B
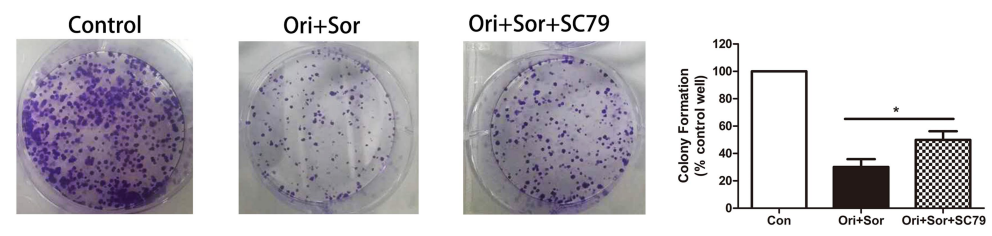

C
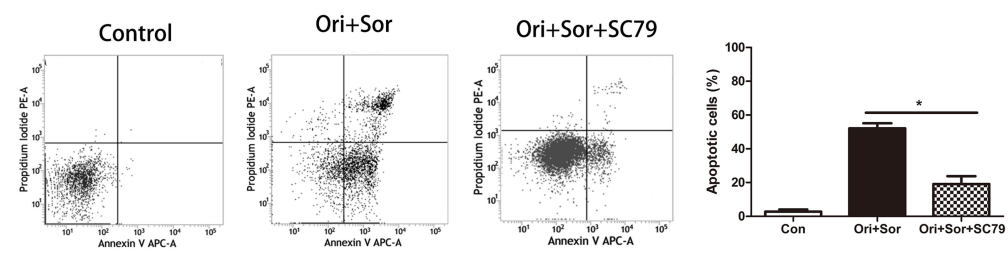

D
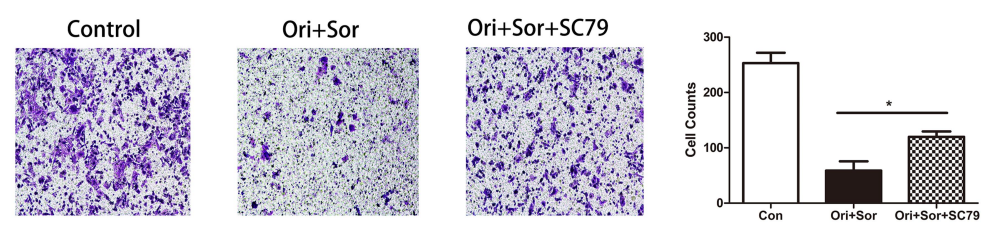

E
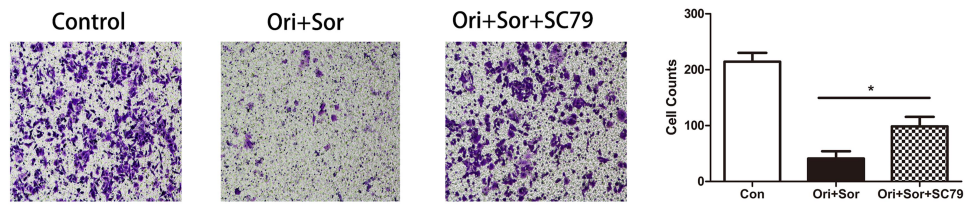

Figure 5 The Akt pathway played an important role in the anticancer effect of combined oridonin and sorafenib treatment. (A) SC79 was used to enhance the phosphorylation of Akt after cotreatment with oridonin and sorafenib, which partially restored the levels of proteins associated with proliferation, apoptosis and EMT. (B) Colony formation assays showed that SC79 treatment restored the proliferation that was inhibited by combined oridonin and sorafenib treatment. (C) Flow cytometry showed that the enhanced apoptosis induction caused by combined oridonin and sorafenib treatment was mitigated by SC79 treatment. (D and E) Reactivation of AKT after SC79 treatment restored cell migration and invasion that had been impaired by cotreatment with the two drugs. (The data represent the mean \pm SD of three independent experiments. *Indicates $\mathrm{p}<0.05$ compared with the combination group; Ori + Sor indicates combined oridonin and sorafenib treatment).

and complementary actions of different pharmacological mechanisms, which is one of the common treatments for cancers. Various studies have shown that the combination of Chinese herbal medicines with sorafenib has synergistic anticancer effects. For instance, the combination of wogonin and sorafenib suppressed HCC through apoptosis and autophagy inhibition; ${ }^{20}$ cotreatment with tanshinone IIA and sorafenib exerted synergistic anticancer effects on HCC ${ }^{21}$ 20(s)-Ginsenoside Rg3 combined with sorafenib inhibited HCC by modulating the PTEN/AKT signaling pathway; ${ }^{22}$ and emodin sensitized HCC cells to the antitumor effect of sorafenib via cholesterol metabolism inhibition. ${ }^{23}$ Our results revealed that cotreatment with the two drugs inhibited proliferation and induced apoptosis in vitro and in vivo. In addition, combined treatment mitigated cell migration and invasion and inhibited EMT. These results indicated that the combination of the two drugs may be a therapeutic strategy for HCC.

Intrinsic resistance to sorafenib caused by the genetic heterogeneity of HCC and acquired resistance developed during sorafenib treatment are the main challenges to improving the therapeutic effect. ${ }^{24}$ The major mechanisms responsible for sorafenib resistance are not completely understood at present. Possible related factors include EGFR activation, epithelial-mesenchymal transition, autophagy, hypoxia, oxidative stress, and activation of escape pathways via the MAPK cascade. ${ }^{25}$ A previous study reported that activation of the PI3K/Akt pathway is a compensatory mechanism to offset sorafenib-induced cell death. ${ }^{26,27}$ In addition, the combination of sorafenib with other drugs might contribute to the suppression of the NF$\mathrm{kB}$ pathway, although sorafenib alone had limited effects on NF-kB pathway inhibition in HCC. ${ }^{13}$ Moreover, MAPK pathways, including JNK, p38 and ERK, are major targets of sorafenib and important in regulating oncological properties. ${ }^{28-30}$ Therefore, we examined the regulatory effect of the Akt, NF-kB, and MAPK pathways after cotreatment. We first found that oridonin decreased the phosphorylation of p65 and inhibited the NF-kB pathway, but sorafenib alone and the combination of the two drugs 

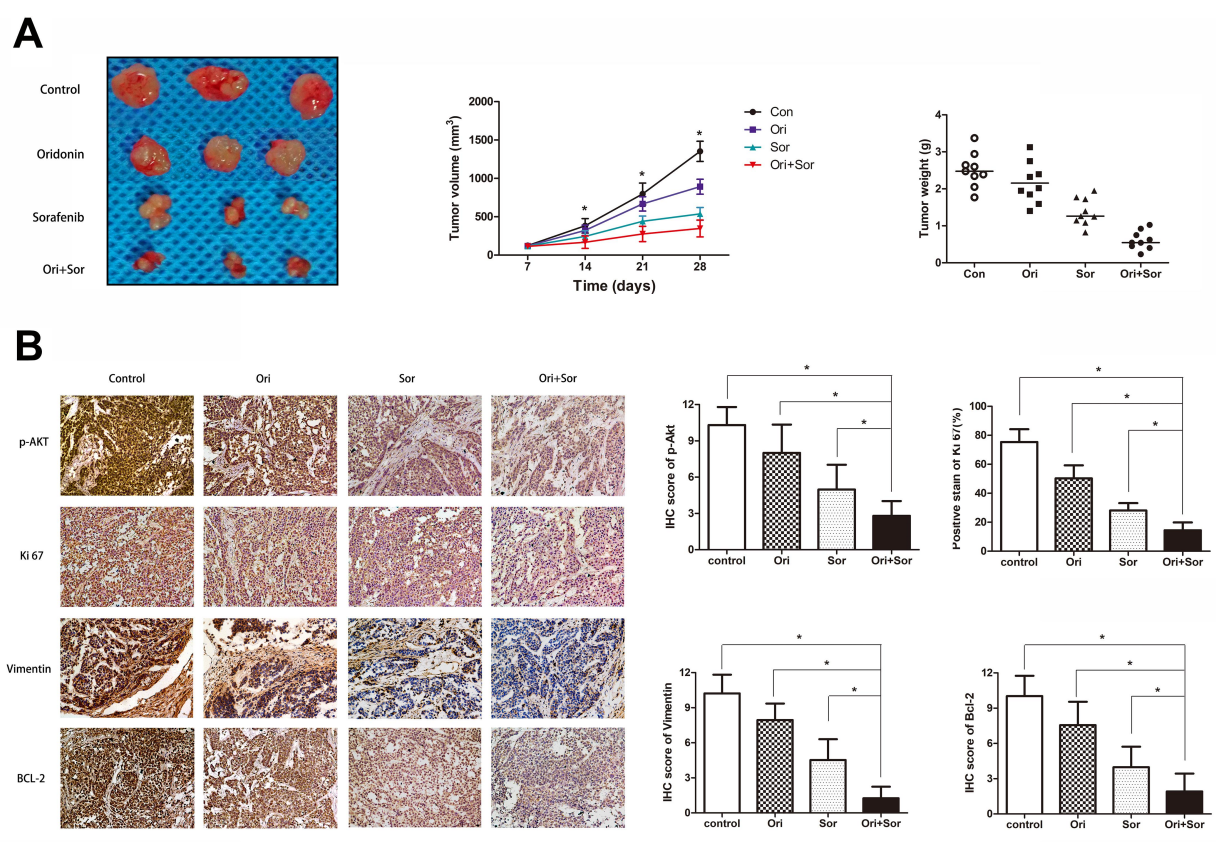

Figure $\mathbf{6}$ The anticancer effect of the combination of oridonin and sorafenib against HCC in vivo. (A) A representative photograph shows the subcutaneous tumors from mice treated with oral administration of vehicle, oridonin, sorafenib and of the combination of the two drugs. Tumor growth curves were prepared by measuring the tumor volumes every week. Tumor weights were recorded after the mice were killed. (B) Representative IHC photographs and stain scores/percentages show the expression levels of $\mathrm{p}$-Akt, Ki67, Vimentin and $\mathrm{Bcl}-2$ in the subcutaneous tumors of mice. (The data represent the mean \pm SD of three independent experiments. $*$ Indicates $\mathrm{p}<0.05$ compared with the combination group; Ori indicates oridonin; Sor indicates sorafenib; Ori + Sor indicates combined oridonin and sorafenib treatment).

did not show significant inhibitory effects. This result indicated that NF-kB might not be a target of the cotreatment. Similar results were observed in the MAPK signaling pathway. Although sorafenib alone inhibited the JNK, p38, and ERK pathways, the combination of the two drugs did not further suppress these signaling pathways. However, oridonin alone inhibited the phosphorylation of Akt, which was consistent with the findings of previous studies in esophageal squamous cell carcinoma and oral cancer. ${ }^{31,32}$ The novel findings in our study are that the cotreatmentinduced inhibition of Akt was superior to that of either drug alone, and the inhibited oncological properties due to cotreatment were reversed after we restored Akt activation by adding SC79 (an Akt activator). These results further confirmed our hypothesis that the combination of oridonin and sorafenib inhibited HCC progression through the Akt pathway. Consistent with our study, previous studies have demonstrated that inhibition of the Akt pathway contributes to enhancing the therapeutic effect of sorafenib in HCC. For instance, Zhai et al demonstrated that bufalin reversed sorafenib resistance through Akt inhibition in HCC. ${ }^{33}$ Our results also showed that cotreatment with oridonin and sorafenib promoted the transition of HCC cells to epithelial cells. Different studies have demonstrated that EMT may be involved in sorafenib resistance mechanisms and compared with epithelial cells, mesenchymal HCC cells are resistant to sorafenib. ${ }^{34,35}$ Although it remains unclear whether combination treatment induced an epithelial transition increased the sensitivity to sorafenib, we demonstrated that EMT participated in the synergistic inhibitory effect of the combination treatment on HCC. Further studies investigating the association between EMT and cotreatment are needed.

\section{Conclusion}

To the best of our knowledge, this is the first study to investigate the synergistic effect of oridonin and sorafenib in HCC. Our research demonstrated that combined oridonin and sorafenib treatment synergistically inhibited HCC progression in vitro and in vivo through the Akt pathway but not the NF-kB or MAPK pathway.

However, there are still some limitations to this study. First, the inhibition of the Akt pathway might be insufficient to elucidate the synergistic inhibitory effect of oridonin and sorafenib cotreatment because the crosstalk associated with HCC progression is complex. Further studies to explore the mechanisms of combined oridonin and sorafenib treatment would be of great value for managing 
hepatocellular carcinoma. Second, a clinical trial is needed to confirm the actual efficacy of the combination treatment before it becomes a therapeutic candidate.

\section{Disclosure}

The authors report no conflicts of interest for this work.

\section{References}

1. Bray F, Ferlay J, Soerjomataram I, Siegel RL, Torre LA, Jemal A. Global cancer statistics 2018: GLOBOCAN estimates of incidence and mortality worldwide for 36 cancers in 185 countries. CA Cancer J Clin. 2018;68(6):394-424. doi:10.3322/caac.21492

2. Liu K, Chen Y, Wu X, et al. Laparoscopic liver re-resection is feasible for patients with posthepatectomy hepatocellular carcinoma recurrence: a propensity score matching study. Surg Endosc. 2017;31 (11):4790-4798. doi:10.1007/s00464-017-5556-3

3. Llovet JM, Ricci S, Mazzaferro V, et al. Sorafenib in advanced hepatocellular carcinoma. $N$ Engl J Med. 2008;359(4):378-390. doi:10.1056/NEJMoa0708857

4. Casadei Gardini A, Faloppi L, De Matteis S, et al. Metformin and insulin impact on clinical outcome in patients with advanced hepatocellular carcinoma receiving sorafenib: validation study and biological rationale. Eur $J$ Cancer. 2017;86:106-114. doi:10.1016/j. ejca.2017.09.003

5. Di Costanzo GG, Casadei Gardini A, Marisi G, et al. Validation of a simple scoring system to predict sorafenib effectiveness in patients with hepatocellular carcinoma. Target Oncol. 2017;12(6):795-803. doi:10.1007/s11523-017-0522-5

6. Brunetti O, Gnoni A, Licchetta A, et al. Predictive and prognostic factors in HCC patients treated with sorafenib. Medicina (Kaunas). 2019;55(10):707.

7. Cheng AL, Kang YK, Chen Z, et al. Efficacy and safety of sorafenib in patients in the Asia-Pacific region with advanced hepatocellular carcinoma: a Phase III randomised, double-blind, placebo-controlled trial. Lancet Oncol. 2009;10(1):25-34. doi:10.1016/S1470-2045(08) 70285-7

8. Bai N, He K, Zhou Z, et al. Ent-kaurane diterpenoids from Rabdosia rubescens and their cytotoxic effects on human cancer cell lines. Planta Med. 2010;76(2):140-145. doi:10.1055/s-0029-1186002

9. Wang H, Ye Y, Chu JH, Zhu GY, Fong WF, Yu ZL. Proteomic and functional analyses reveal the potential involvement of endoplasmic reticulum stress and alpha-CP1 in the anticancer activities of oridonin in HepG2 cells. Integr Cancer Ther. 2011;10(2):160-167. doi:10.1177/1534735410383171

10. Dong X, Liu F, Li M. Inhibition of nuclear factor kappaB transcription activity drives a synergistic effect of cisplatin and oridonin on HepG2 human hepatocellular carcinoma cells. Anticancer Drugs. 2016;27(4):286-299. doi:10.1097/CAD.0000000000000329

11. Park H, Jeong YJ, Han NK, Kim JS, Lee HJ. Oridonin enhances radiation-induced cell death by promoting DNA damage in non-small cell lung cancer cells. Int J Mol Sci. 2018;19(8):2378. doi:10.3390/ ijms 19082378

12. Zheng W, Zhou CY, Zhu XQ, et al. Oridonin enhances the cytotoxicity of 5-FU in renal carcinoma cells by inducting necroptotic death. Biomed Pharmacother. 2018;106:175-182. doi:10.1016/j.biopha. 2018.06.111

13. Tan W, Luo X, Li W, et al. TNF-alpha is a potential therapeutic target to overcome sorafenib resistance in hepatocellular carcinoma. EBioMedicine. 2019;40:446-456. doi:10.1016/j.ebiom.2018.12.047

14. Wang H, Ye Y, Pan SY, et al. Proteomic identification of proteins involved in the anticancer activities of oridonin in HepG2 cells. Phytomedicine. 2011;18(2-3):163-169. doi:10.1016/j.phymed.2010. 06.011
15. Liu X, Kang J, Wang H, Huang T. Mitochondrial ROS contribute to oridonin-induced HepG2 apoptosis through PARP activation. Oncol Lett. 2018;15(3):2881-2888. doi:10.3892/ol.2017.7665

16. Huang J, Wu L, Tashiro S, Onodera S, Ikejima T. Reactive oxygen species mediate oridonin-induced HepG2 apoptosis through p53, MAPK, and mitochondrial signaling pathways. J Pharmacol Sci. 2008;107(4):370-379. doi:10.1254/jphs.08044FP

17. Luo D, Yi Y, Peng K, et al. Oridonin derivatives as potential anticancer drug candidates triggering apoptosis through mitochondrial pathway in the liver cancer cells. Eur $J$ Med Chem. 2019;178:365-379. doi:10.1016/j.ejmech.2019.06.006

18. Li D, Cai H, Jiang B, et al. Synthesis of spirolactone-type diterpenoid derivatives from kaurene-type oridonin with improved antiproliferative effects and their apoptosis-inducing activity in human hepatoma Bel-7402 cells. Eur J Med Chem. 2013;59:322-328. doi:10.1016/j. ejmech.2012.11.002

19. Wilhelm S, Carter C, Lynch M, et al. Discovery and development of sorafenib: a multikinase inhibitor for treating cancer. Nat Rev Drug Discov. 2006;5(10):835-844. doi:10.1038/nrd2130

20. Rong LW, Wang RX, Zheng XL, et al. Combination of wogonin and sorafenib effectively kills human hepatocellular carcinoma cells through apoptosis potentiation and autophagy inhibition. Oncol Lett. 2017;13(6):5028-5034. doi:10.3892/ol.2017.6059

21. Chiu CM, Huang SY, Chang SF, Liao KF, Chiu SC. Synergistic antitumor effects of tanshinone IIA and sorafenib or its derivative SC-1 in hepatocellular carcinoma cells. Onco Targets Ther. 2018;11:1777-1785. doi:10.2147/OTT.S161534

22. Lu M, Fei Z, Zhang G. Synergistic anticancer activity of 20 (S)-Ginsenoside $\operatorname{Rg} 3$ and Sorafenib in hepatocellular carcinoma by modulating PTEN/Akt signaling pathway. Biomed Pharmacother. 2018;97:1282-1288. doi:10.1016/j.biopha.2017.11.006

23. Kim YS, Lee YM, Oh TI, et al. Emodin sensitizes hepatocellular carcinoma cells to the anti-cancer effect of sorafenib through suppression of cholesterol metabolism. Int J Mol Sci. 2018;19(10):3127. doi:10.3390/ijms 19103127

24. Niu L, Liu L, Yang S, Ren J, Lai PBS, Chen GG. New insights into sorafenib resistance in hepatocellular carcinoma: responsible mechanisms and promising strategies. Biochim Biophys Acta Rev Cancer. 2017;1868(2):564-570. doi:10.1016/j.bbcan.2017.10.002

25. Zhu YJ, Zheng B, Wang HY, Chen L. New knowledge of the mechanisms of sorafenib resistance in liver cancer. Acta Pharmacol Sin. 2017;38(5):614-622. doi:10.1038/aps.2017.5

26. Chen KF, Chen HL, Tai WT, et al. Activation of phosphatidylinositol 3-kinase/Akt signaling pathway mediates acquired resistance to sorafenib in hepatocellular carcinoma cells. J Pharmacol Exp Ther. 2011;337(1):155-161. doi:10.1124/jpet.110.175786

27. Wu CH, Wu X, Zhang HW. Inhibition of acquired-resistance hepatocellular carcinoma cell growth by combining sorafenib with phosphoinositide 3-kinase and rat sarcoma inhibitor. J Surg Res. 2016;206 (2):371-379. doi:10.1016/j.jss.2016.08.014

28. Wang C, Jin H, Gao D, et al. Phospho-ERK is a biomarker of response to a synthetic lethal drug combination of sorafenib and MEK inhibition in liver cancer. $J$ Hepatol. 2018;69(5):1057-1065. doi:10.1016/j.jhep.2018.07.004

29. Park GB, Choi Y, Kim YS, Lee HK, Kim D, Hur DY. ROS-mediated $\mathrm{JNK} / \mathrm{p} 38$-MAPK activation regulates Bax translocation in Sorafenib-induced apoptosis of EBV-transformed B cells. Int J Oncol. 2014;44(3):977-985. doi:10.3892/ijo.2014.2252

30. Nguyen TV, Sleiman M, Moriarty T, Herrick WG, Peyton SR. Sorafenib resistance and JNK signaling in carcinoma during extracellular matrix stiffening. Biomaterials. 2014;35(22):5749-5759. doi:10.1016/j.biomaterials.2014.03.058

31. Song M, Liu X, Liu K, et al. Targeting AKT with oridonin inhibits growth of esophageal squamous cell carcinoma in vitro and patient-derived xenografts in vivo. Mol Cancer Ther. 2018;17 (7):1540-1553. doi:10.1158/1535-7163.MCT-17-0823 
32. Yang J, Ren X, Zhang L, Li Y, Cheng B, Xia J. Oridonin inhibits oral cancer growth and PI3K/Akt signaling pathway. Biomed Pharmacother. 2018;100:226-232. doi:10.1016/j.biopha.2018.02.011

33. Zhai B, Hu F, Yan H, et al. Bufalin reverses resistance to sorafenib by inhibiting Akt activation in hepatocellular carcinoma: the role of endoplasmic reticulum stress. PLoS One. 2015;10(9):e0138485. doi:10.1371/journal.pone.0138485

34. van Zijl F, Mall S, Machat G, et al. A human model of epithelial to mesenchymal transition to monitor drug efficacy in hepatocellular carcinoma progression. Mol Cancer Ther. 2011;10(5):850-860. doi:10.1158/1535-7163.MCT-10-0917
35. van Malenstein H, Dekervel J, Verslype C, et al. Long-term exposure to sorafenib of liver cancer cells induces resistance with epithelial-tomesenchymal transition, increased invasion and risk of rebound growth. Cancer Lett. 2013;329(1):74-83. doi:10.1016/j.canlet. 2012.10.021

\section{Publish your work in this journal}

Cancer Management and Research is an international, peer-reviewed open access journal focusing on cancer research and the optimal use of preventative and integrated treatment interventions to achieve improved outcomes, enhanced survival and quality of life for the cancer patient.
The manuscript management system is completely online and includes a very quick and fair peer-review system, which is all easy to use. Visit http://www.dovepress.com/testimonials.php to read real quotes from published authors. 